\title{
Flagellum Mutants of Chlamydomonas reinhardii
}

\author{
By ANNE MCVITTIE* \\ Department of Biophysics, University of London King's College, \\ 26-29 Drury Lane, London, W.C. 2
}

(Accepted for publication I March I972)

\begin{abstract}
SUMMARY
Mutants blocked in different stages of flagellar development were isolated and examined by light and electron microscopy. Flagellum-less mutants had normal basal bodies but lacked the flagellar shaft and showed abnormalities in the transitional region. Stumpy mutants had very short flagellar shafts in which electrondense material, sometimes arranged in sheet-like arrays, accumulated in the space between the outer tubules and the distended flagellar membrane. Short-flagellum mutants also showed abnormalities in the flagellar shaft; abnormally spaced radial spokes were seen in negatively stained frayed flagella of one of these mutants. The flagellum lengths and flagellar regeneration characteristics of two long-flagellum mutants with defective length control were determined; length was highly variable with some abnormally long flagella, and regeneration was slow and asynchronous.
\end{abstract}

\section{INTRODUCTION}

Chlamydomonas reinhardii is one of the few unicellular eukaryotes suitable for genetic analysis and therefore offers promising material for studying the developmental genetics of cell organelles. The flagellum is a convenient organelle for this kind of investigation since mutants with altered flagella can be readily isolated and much is already known about flagellar structure. Analysis of mutants identifies the genes concerned with various aspects of the structure, function and development of the organelle: a full characterization of each mutant establishes the role of the corresponding wild-type gene product. These ideas were discussed more fully by Randall, Warr, Hopkins \& McVittie (I964), Warr, McVittie, Randall \& Hopkins (I966), Randall, Cavalier-Smith, McVittie, Warr \& Hopkins (1967) and Randall (1969).

Mutants of Chlamydomonas reinhardii isolated on the basis of non-motility or partially impaired motility include strains with structurally defective flagella (Randall et al. I964; Warr et al. 1966). These strains have disorganized central tubules and were termed ' $9+0$ ' to distinguish them from the wild-type in which flagella are of the ' $9+2$ ' type (nine outer doublet tubules and two central singlet tubules). In this paper, which reports the isolation and characterization of further flagellum mutants, additional classes of mutant are described. These include three classes of developmental mutant producing incomplete, morphologically abnormal flagella, and one class (long-flagellum mutants) defective in the control of flagellum length and elongation rate. The mutants have been characterized by light and electron microscopy and regeneration studies have been carried out on the long-flagellum mutants. Genetic analysis of the mutants is described elsewhere (McVittie, 1972).

\footnotetext{
* Present address: John Innes Institute, Norwich, NOR 7oF.
} 


\section{METHODS}

Strains. The wild-type strains of Chlamydomonas reinhardii 32C and 32D (mating types plus and minus respectively) were obtained from the Cambridge Culture Collection of Algae and Protozoa. The paralysed mutants $p f_{I-1 O}, 12-15,15 A, I 6-19,19 A$ and 20 were obtained from Professor R. P. Levine, Harvard University. The origin of $p f_{I} 8 A$ to $D, p f 19 B$ to $D$ and pf $20 A$ was described by Warr et al. (I966).

Media and culture conditions. Cultures were grown on a simple inorganic medium (Warr et al. 1966) in a light box maintained at $25^{\circ} \mathrm{C}$ under continuous illumination of $500 \mathrm{ft}$-candles. Ammonium nitrate was omitted from the growth medium to give nitrogen-free medium (NFM).

Mutagenesis. The methods for acridine orange (AO) and hydroxylamine (HA) treatment were those of Warr et al. (I966). Two methods were used for mutagenesis by $N$-methyl- $N^{\prime}$ nitro- $N$-nitrosoguanidine (NG, Koch-Light Laboratories, Colnbrook, Buckinghamshire). Organisms (32C) growing exponentially were plated on semisolid medium containing 0.5 , $\mathrm{I} \cdot 0$ or $2.0 \mu \mathrm{g} \mathrm{NG} / \mathrm{ml}$ and mutant colonies picked after 5 to 8 days of incubation. A freshly made solution of NG in sterile water was added to the agar just before pouring. The second method involved spreading $0.1 \mathrm{ml}$ of a late exponential-phase culture of $32 \mathrm{C}$ containing $\mathrm{I}-2 \times \mathrm{IO}^{6}$ organisms $/ \mathrm{ml}$ on medium containing $0.5 \mu \mathrm{g} \mathrm{NG} / \mathrm{ml}$. After 2 days of incubation each plate was harvested separately in distilled water and the suspensions were diluted and plated on semisolid medium to give about Ioo cells/plate. Mutants with impaired motility were distinguished from wild-type by their smaller, more heaped colonies (Warr et al. I966). Only one mutant of a given phenotype was isolated from each suspension to ensure an independent origin for each mutant; all mutants were cloned before being characterized.

Light and electron microscopy. Phase-contrast photomicrographs were taken on a Zeiss Photomicroscope using Ilford Pan F film. For thin sectioning the algae were fixed in suspension for $20 \mathrm{~min}$ at room temperature in $\mathrm{I} \%(\mathrm{w} / \mathrm{v})$ osmium tetroxide in $0.14 \mathrm{M}$-veronal acetate buffer at $\mathrm{pH} 7 \cdot 4$. The suspensions were centrifuged at $500 \mathrm{~g}$ for $5 \mathrm{~min}$ and the pellets dehydrated in acetone and embedded in Araldite (Luft, 196I). Silver sections were stained with saturated uranyl acetate and lead citrate and examined in a Siemens Elmiskop I operating at $80 \mathrm{kV}$ and using a $50 \mu \mathrm{m}$ objective aperture.

Frayed flagella for negative staining with $0.5 \%(\mathrm{w} / \mathrm{v})$ aqueous uranyl acetate were obtained by the method of Hopkins (1970). Organisms were suspended in a low ionic strength buffer (T/EDTA) at $\mathrm{pH} 7 \cdot 7$ for 30 to $60 \mathrm{~min}$ at $4{ }^{\circ} \mathrm{C}$; this caused disruption and swelling of the flagellar membrane (Jacobs \& McVittie, 1970). They were then subjected to ultrasonic vibration for Io s.

Flagellum length measurements and regeneration experiments. A map-measuring instrument was used to measure tracings of flagella made from phase-contrast negatives projected to give a final magnification of 7000 . For regeneration experiments organisms were deflagellated by adding $0.1 \mathrm{ml}$ of $0.3 \%(\mathrm{v} / \mathrm{v})$ glacial acetic acid to $0.9 \mathrm{ml}$ of a concentrated cell suspension in NFM. After 3 min (time zero) the mixture was diluted tenfold, centrifuged at $500 \mathrm{~g}$ for $5 \mathrm{~min}$ and the algae resuspended in $4 \mathrm{ml} \mathrm{NFM}$. The suspensions were placed in the light box and intermittently agitated gently. Samples taken at intervals were fixed in osmium tetroxide vapour and photographed. In each sample, 200 organisms were scored for the presence of flagella to determine the percentage of flagellated organisms (F). Flagellum lengths of 25 flagellated algae (50 flagella) were usually measured to obtain the mean length $\left(M_{\mathrm{f}}\right)$. In the early samples where some organisms still lacked flagella the mean for the whole population 
Table I. Nitrosoguanidine mutagenesis

$\begin{array}{ccccc}\text { Expt } & \begin{array}{c}\text { NG } \\ \text { concentration } \\ (\mu \mathrm{g} / \mathrm{ml})\end{array} & \begin{array}{c}\text { Survival } \\ (\%)\end{array} & \begin{array}{c}\text { Flagellum } \\ \text { mutants } \\ \text { isolated }\end{array} & \begin{array}{c}\text { No. of } \\ \text { colonies } \\ \text { screened }\end{array} \\ \text { I } & 5 & <0 . \mathrm{I} & 0 & 0 \\ & 2 & \mathbf{I} 4 & + & 173 \\ 2 & 2 & 17 & 0 & 123 \\ & \text { I } & 20 & + & 146 \\ & \text { O.5 } & 67 & + & 489 \\ & \text { O.I } & 100 & 0 & \text { c. } 740 \\ 3 & \text { O.5 } & 75 & + & - \\ 4 & \text { O.5 } & 89 & + & - \\ 5 & 0.5 & 68 & + & - \\ 6 & 0.5 & 63 & + & -\end{array}$

$\left(M_{\mathrm{p}}\right)$ was obtained by the formula $M_{\mathrm{p}}=M_{\mathrm{f}} \times(\mathrm{F} / \mathrm{IOO})$. The $M_{\mathrm{p}}$ values were then plotted against time.

\section{RESULTS}

\section{Nitrosoguanidine mutagenesis and isolation of mutants}

It was possible to isolate motility mutants directly from platings of wild-type organisms on semisolid medium containing low and relatively non-lethal concentrations of NG (Table I, Expts I and 2). The colonies obtained on these plates were very heterogeneous in size and appearance, but a high proportion of those picked on the basis of their small size and heaped appearance constituted pure clones of mutant organisms as determined by subsequent platings on semisolid medium. In Expt I, I 2 of the 16 colonies picked were pure mutant clones, while three were mixtures of mutant and wild-type cells and one appeared to contain only wild-type cells. These I6 colonies were picked from a total of only I 73 and yielded six relatively non-leaky motility mutants. The remaining colonies probably contained leaky auxotrophs or leaky motility mutants. In Expt 2, I 3 colonies were picked from a total of 635 (on the plates containing 0.5 and $\mathrm{I} \cdot 0 \mu \mathrm{g} \mathrm{NG} / \mathrm{ml}$ ) and yielded five motility mutants. As before, most of the picked colonies were pure mutant clones.

The remaining $26 \mathrm{NG}$-induced mutants described here were isolated from Expts 3 to 6 (Table I) using the second method of mutagenesis (see Methods). About Iooo colonies from each of 32 suspensions of NG-treated cells were examined. One hundred and two further strains isolated in these experiments were discarded after a preliminary investigation. They included strains which produced small colonies for some reason other than impaired motility, palmella mutants (Warr et al. I966), a few mutants in phenotypic class 4 and a few leaky mutants in classes 6,7 and 8 (Table 2).

In Expts 3 to 6 colonies were initially picked at $32^{\circ} \mathrm{C}$ in an attempt to isolate temperaturesensitive motility mutants. They were subsequently tested at 32 and $25^{\circ} \mathrm{C}$ (or at $20^{\circ} \mathrm{C}-\mathrm{Expt}$ 6). Five of the total of $\mathrm{I} 28$ strains were probably temperature-sensitive (mutant only at $32^{\circ} \mathrm{C}$ ) and were also leaky. Three of these were abnormally swimming mutants, one was a shortflagellum mutant and one was probably flagellum-less, but leakiness made them difficult to characterize.

In addition to the 37 mutants induced by $\mathrm{NG}$, five mutants were isolated following $\mathrm{AO}$ treatment, I3 after treatment with HA and three arose spontaneously thus giving a total of 58. Organisms treated with AO or HA were enriched by further growth before being scored 
Table 2. Flagellum mutants of Chlamydomonas reinhardii

Phenotype
1. Straight flagella $(9+0)$
2. Curved flagella
3. Flagella in various
positions
4. Abnormal swimming
5. Swollen flagella
6. Flagellum-less
7. Stumpy
8. Short flagella
9. Long flagella

Newly isolated mutants

8 ( $p f_{I} 5 B, p f I 8 E, F, p f r g E$, pf $20 B$ to $E$ )

2 (pfI $A$ and $B$ )

2

6

5

8

6

$9\left(p f_{7} A\right.$ and $B, p f 8 A$ to $D$, pf $21, S I 6, H A 24)$

2 (lf 1, lf 2$)$

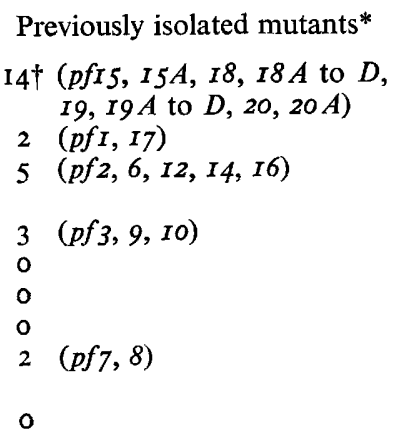

No. of loci

known

4

2

5

2

Unknown

Unknown

Unknown

3

2

* See Ebersold, Levine, Levine \& Olmsted (1962).

$\uparrow$ See Warr et al. (1966).

for mutants. It was therefore impossible to determine the mutation frequency and to.establish whether the mutants had been induced or had arisen spontaneously.

Many of the mutants were tested for segregation at meiosis in crosses to wild-type or other strains; ten or more tetrads were analysed in each cross. Twenty of the 23 NG-induced strains tested showed 2:2 segregation of normal to abnormal motility indicating mutation in a single gene, while the remaining three strains gave a mixture of parental and nonparental ditype and tetratype tetrads with respect to normal versus abnormal motility and large versus small colony size on semisolid medium. Thus each of these three strains carried a mutation having some effect on colony size (probably because of slow growth) in addition to the mutation affecting motility. In each case the latter single mutation was isolated from the back-cross for subsequent study. Nine of the strains isolated after AO and HA treatment were tested and all gave 2:2 segregation in all tetrads.

Types of mutant isolated. Nine main phenotypes have been distinguished among the flagellum mutants isolated here and previously (Table 2). Some of the strains isolated after AO and HA treatment and listed in the first column appeared in an earlier table (Warr et al. I966) but they have been included here for completeness. Except for some of the mutants with straight flagella, the strains listed in the second column were all isolated by Dr R. A. Lewin. The mutants belonging in some of the classes are given in brackets.

Descriptions of the first five phenotypic classes were given in previous publications (Warr et al. 1966; Randall et al. 1967). It was confirmed by electron microscopy that the newly isolated straight-flagellum mutants $p f_{15} B, p f_{I} 8 F$ and $p f 2 O B$ had the $9+0$ structure with disorganized central tubules characteristic of mutants at these loci. Strains $p f 20 B$ to $E$ resembled $p f 20$ in having a small proportion of spinning and swimming cells. Mutants in classes 6 to 8 were described briefly by McVittie (1969) and some results of work with longflagellum mutants were reported by Randall et al. (1967); further details are given in the following sections. Class Io includes mutants which may be of the palmella type $\left(p f_{4}, 5\right.$ and 13) and strains which were difficult to characterize.

Flagellum-less mutants. These strains lacked externally visible flagella even under conditions which gave maximum flagellation in other strains (see section on short-flagellum mutants). Electron-microscopic examination showed that the basal body and transitional region of the flagellum were present, but that the flagellum proper, or flagellar shaft, was almost completely absent (Fig. I $a$ ). The flagellum ended before emerging from the collar, a 

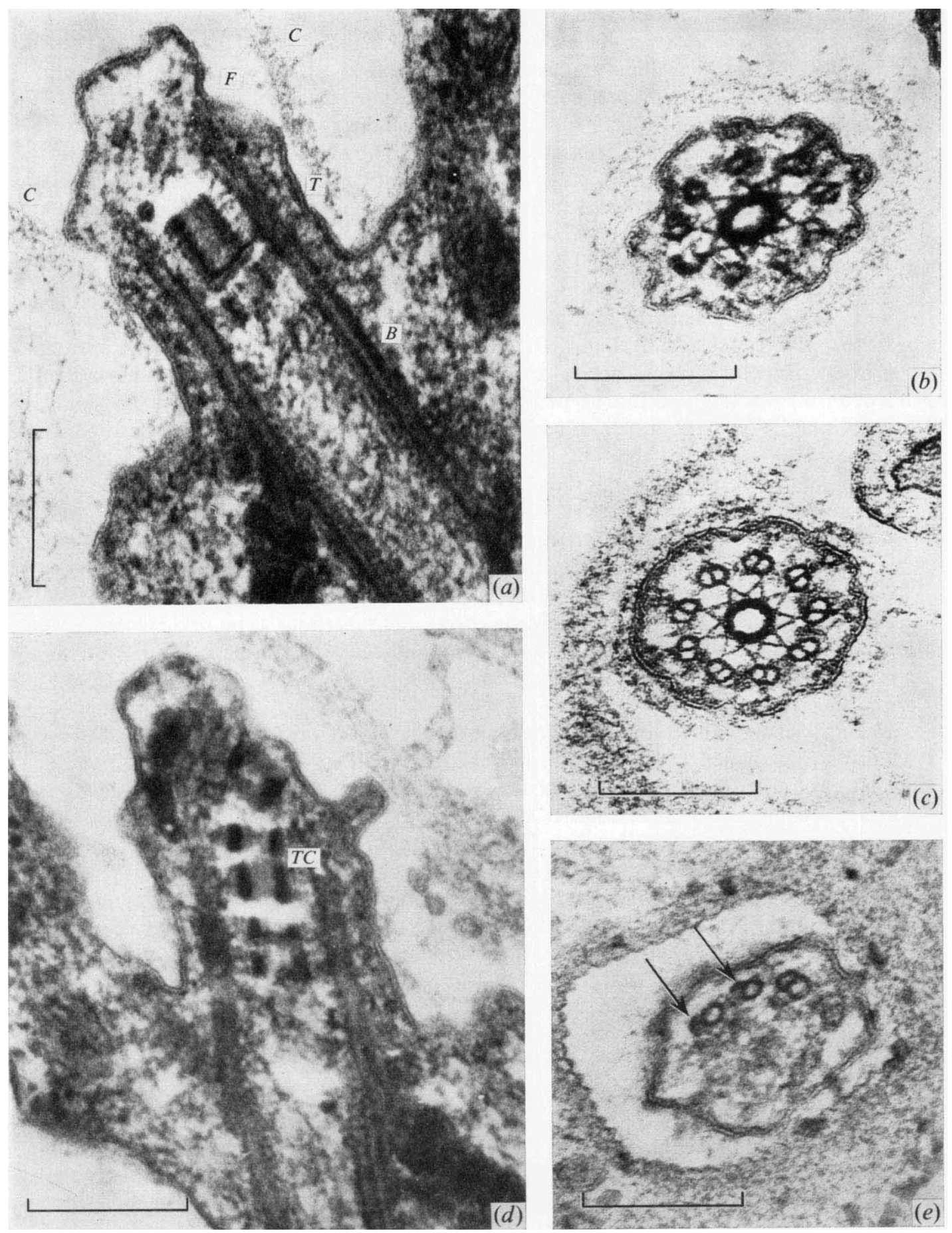

Fig. I. Electron micrographs of thin sections. Scale marks represent $0.2 \mu \mathrm{m}$. (a) Flagellum-less mutant $S_{3}$. Longitudinal section showing basal body $(B)$, transitional region $(T)$ and very short flagellar shaft $(F)$; wall collar $(C)$. (b) Flagellum-less mutant $S_{3}$. Cross-section through transitional region. (c) Wild-type; cross-section through transitional region. (d) Flagellum-less mutant $N G_{30}$; longitudinal section showing abnormal transitional region cylinder $(T C)$. (e) Flagellum-less mutant NG6; cross-section through flagellar shaft showing one complete doublet tubule and two A subtubules with incomplete B subtubules (arrows). 
specialized region of the cell wall. (A full description of the fine structure of the flagellar apparatus in Chlamydomonas reinhardii is given by Ringo (1967); his terminology is used here except that flagellar fibres are here called tubules.)

The basal bodies and additional components of the flagellar apparatus (proximal and distal striated fibres, transitional fibres and microtubule bands) appeared normal, but structural abnormalities were evident in the transitional region. The nine outer doublet tubules, which are continuous with the triplet tubules of the basal body, usually ended at the same level as the distal end of the transitional-region distal cylinder (Fig. I $a$ ). The doublets showed structural disorganization near their ends. Cross-sections often showed a mixture of complete and incomplete doublets and singlets (Fig. I $b$ ). For comparison, Fig. I $(c)$ shows the appearance of transverse sections in this region of the wild-type.

In most of the mutants the transitional-region cylinders, particularly the distal cylinder, were sometimes abnormal. In Fig. I $(d)$, for instance, the distal cylinder appears to be extended in length and broken; the normal appearance is seen in Fig. I $(a)$. In some of the mutants the outer tubules extended just beyond the transitional region (Fig. I $e$ ) but central tubules have not been seen. The flagellum proper thus usually consisted of a membrane-bounded region extending for a very short distance beyond the transitional region and virtually devoid of tubules. Fig. I $(e)$ also indicates that the development of outer tubules in Chlamydomonas flagella occurs in the same way as in the basal bodies of Paramecium aurelia (Dippell, I968). The A subtubule is formed first as a singlet tubule and the B subtubule gradually extends from it as a curved sheet, so that eventually a doublet tubule is formed.

There was some variation between mutants in the degree of structural disorganization seen in the transitional region, possibly indicating that mutations at more than one genetic locus might be represented among these eight strains. However, genetic analysis of these strains was impossible owing to the apparently obligatory involvement of flagella in the initial stages of mating.

Stumpy mutants. These strains had very short flagella, just visible in the light microscope. Measurements made from electron micrographs showed that the flagellum proper was less than $\mathrm{I} \mu \mathrm{m}$ long. The fine structure of the basal body and transitional region and of the additional components of the flagellar apparatus was normal, but several structural abnormalities were seen in the flagellar shaft. Although normal $9+2$ arrays of well-formed tubules with attached subsidiary components such as arms and spokes (see Hopkins, 1970) were seen (Fig. 2a), there was usually some breakdown of tubule structure and arrangement before the end of the flagellum was reached (Fig. $2 b-d$ ). Central tubules were sometimes completely absent (Fig. 2c). The flagellar membrane was frequently distended giving an enlarged space between it and the outer tubules (Fig. $2 d$ ). There was usually some scattered electron-dense material within the flagellar shaft, especially in the region between the outer tubules and the membrane (Fig. 2a-d).

As in the case of flagellum-less mutants, lack of mating precluded genetic analysis of these six strains and phenotypic heterogeneity indicated that more than one genetic locus might be involved.

Short-flagellum mutants. Preliminary observations were made on organisms grown on agar, harvested in late exponential or early stationary phase and suspended for about $4 \mathrm{~h}$ in distilled water. The percentage of organisms with flagella of measurable length was low, ranging from 3 to $12.5 \%$; the mean lengths of these flagella were about $3 \mu \mathrm{m}$ and the maximum was in the range 4 to $7 \mu \mathrm{m}$, except in the case of strain SI6, which had a mean of about $\mathrm{I} \cdot 5 \mu \mathrm{m}$ and a maximum of only $2 \mu \mathrm{m}$. Many of the organisms classed as lacking measurable flagella had short stumps. 

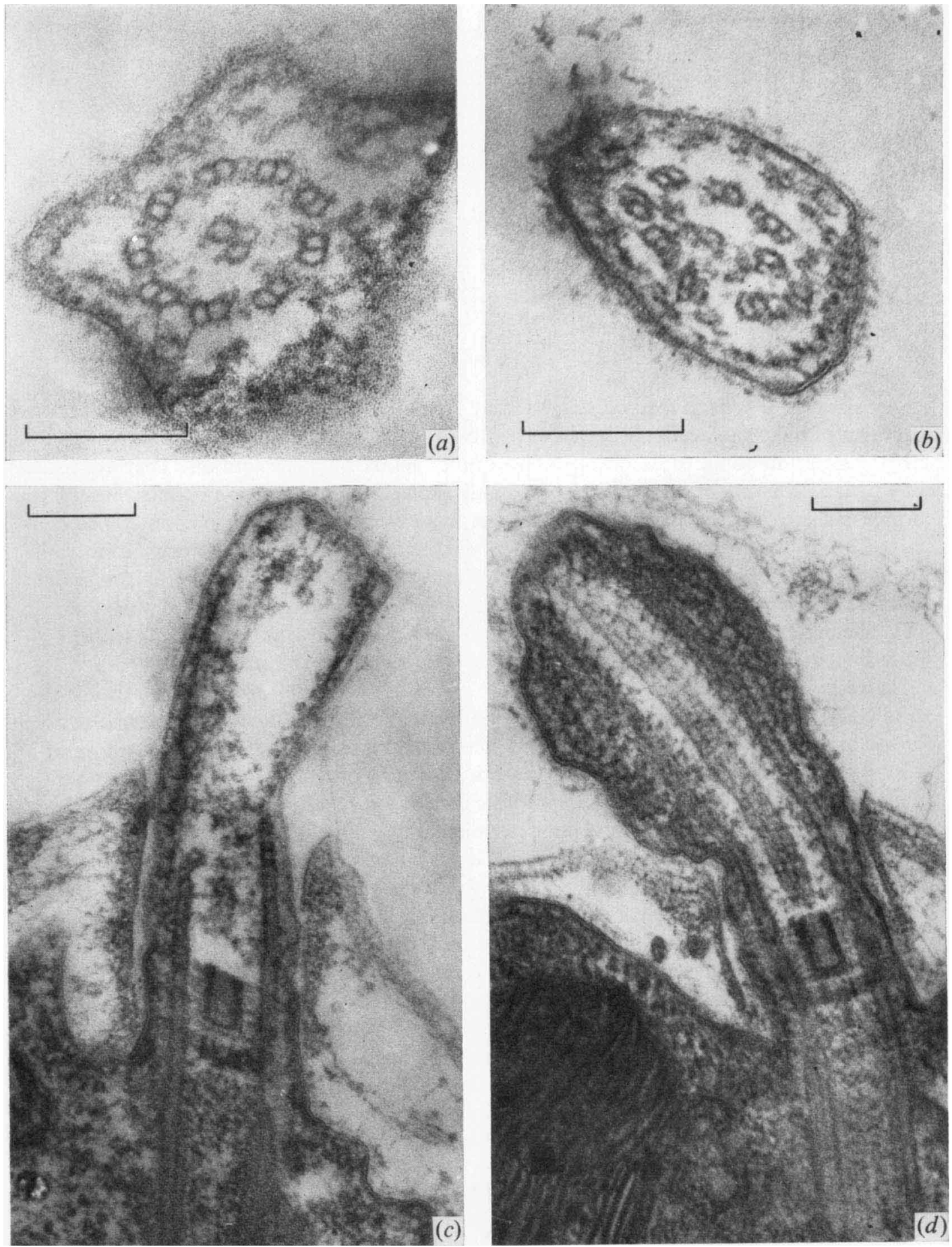

Fig. 2. Electron micrographs of thin sections of stumpy mutant $H A I$. Scale marks represent $0.2 \mu \mathrm{m}$. $(a)$ and $(b)$ Cross-sections through flagellar shaft. $(c)$ and $(d)$ Longitudinal sections. 
Table 3. Maximum flagellum length of short flagellum mutants in NFM

$\begin{array}{lcc}\text { Mutant } & \begin{array}{c}\text { Single organisms } \\ \text { having flagella } \\ (\%)\end{array} & \begin{array}{c}\text { Maximum flagellum } \\ \text { length }(\mu \mathrm{m})\end{array} \\ p f_{7} & 67 & 6 \cdot \mathrm{I} \\ p f_{7} A & 96 & 7 \cdot \mathrm{I} \\ p f 7 B & 83 & 6 \cdot 9 \\ p f 8 & 82 & 6 \cdot 7 \\ p f 8 A & 92 & 9 \cdot 3 \\ p f 8 B & 89 & 7 \cdot 6 \\ p f 8 C & 8 \mathrm{I} & 5 \cdot 7 \\ p f 8 D & 84 & 6 \cdot 4 \\ p f 2 I & 61 & 9 \cdot 6 \\ H A 24 & 84 & 5 \cdot 7\end{array}$

An alternative growth method suggested by Dr D. Starling was found to give higher percentages of fiagellated cells. Cultures were grown for 3 days to a density of about $1 \cdot 5 \times 10^{6}$ organisms $/ \mathrm{ml}$ in $\mathrm{I} 0 \mathrm{ml}$ of liquid medium in tubes gently bubbled with air. They consisted of a variable mixture of single algae and post-division clumps of 4,8 , or more non-flagellated organisms surrounded by the mother wall. In two experiments strains $p f 8 A$ and $p f 8 B$ contained mostly single organisms, $p f 7 A$ many, and $p f 8 D$ and $S_{I} 6$ few single organisms, while the remaining strains had intermediate values. In general, removal of the algae from growth medium and resuspension in NFM or distilled water was found to increase both the number of single organisms and the percentages of flagellated organisms among the singles, although little improvement in the proportion of singles was seen in $p_{7} B, p f_{21}, H_{A 24}$ and $S_{16}$.

Table 3 gives the results of an experiment in which the organisms were resuspended in $2 \mathrm{ml} \mathrm{NFM}$ and bubbled with air in the light box for $\mathrm{I} \cdot 5,4$ or $7 \mathrm{~h}$ before observation. Samples of 200 organisms were used for determining the percentages flagellated (including those with very short flagella), and samples of 50 or more flagellated organisms were used in measuring the maximum flagellum lengths. Except in the case of $p f 8 A$ and $p f 2 I$ maximum lengths were less than $8 \mu \mathrm{m}$. Mean lengths were not determined but would have been considerably lower than the maximum values since there was usually a wide range in length with some very short flagella. They would thus be well below the wild-type value of $10.5 \mu \mathrm{m}$ after $4 \mathrm{~h}$ in NFM (Randall et al. 1967). In any case the mean values would be continuously changing owing to increases in the percentage of single organisms and emergence of new flagella, and to flagellar growth and regression (see below).

Flagellar regression was seen in $p f 7 A$ and $p f 8$, i.e. the proportion of cells with very short flagella was much higher at 7 than at $4 \mathrm{~h}$. It may also have occurred in $p f 7$ and $p f 8 A$. Strain $p f 8 A$ usually contained some cells in which the two flagella were of grossly unequal length (Fig. $3 e$ ), and $p f 8 B$ and $p f 2 I$ also had occasional cells with flagella of unequal length. Strain pf $8 \mathrm{~A}$ was also the only one in which occasional swimming cells and a few spinning cells were seen, the latter presumably being cells with one normal and one short flagellum. The flagella of $p f 2 I$ were completely motionless but those of all the other strains listed in Table 3 showed some twitching. Even when bubbled in NFM, $S_{16} 6$ had only very short stumps or short flagella which showed no twitching.

Thin sections showed that fine structural abnormalities of the flagellar apparatus were confined, in these mutants, to the flagellar shaft. There was a tendency for the tubules to be curved (Fig. $3 c$ ) or occasionally bent (Fig. $3 d$ ). As in stumpy mutants, tubules were sometimes poorly formed and displaced from their normal positions (Fig. $3 a$-compare with the 

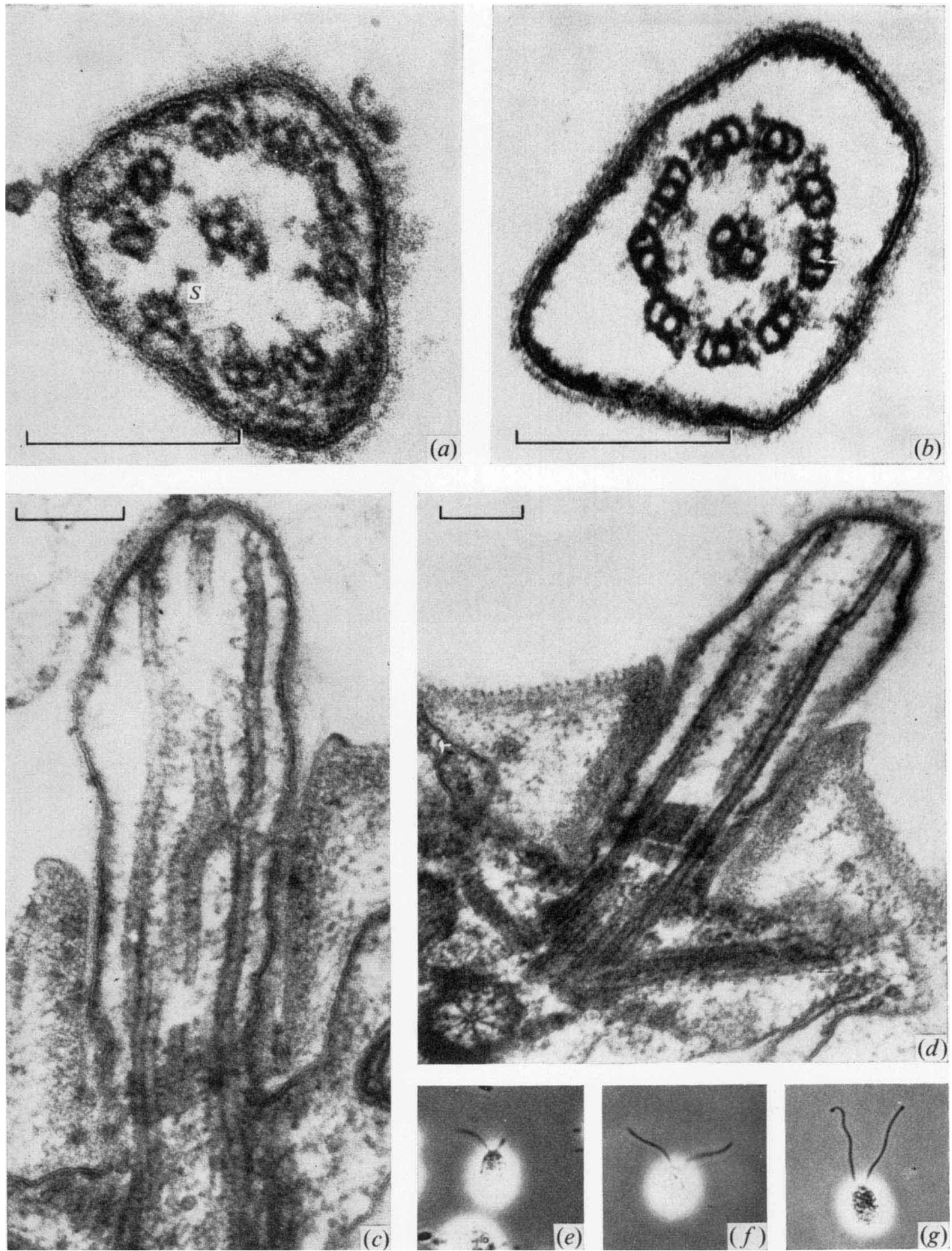

Fig. 3. (a) to $(d)$ Electron micrographs of thin sections. $(e)$ to $(g)$ Phase-contrast photomicrographs. Scale marks represent $0.2 \mu \mathrm{m}$. (a) Short-flagellum mutant $p f 7$. Cross-section through flagellar shaft showing radial spokes (S). (b) Wild-type; cross-section through flagellar shaft. (c) Short-flagellum mutant $p f 2 r$; longitudinal section. (d) Short-flagellum mutant $p f 8 D$; longitudinal section. (e) Shortflagellum mutant $p f 8 A$ showing unequal length flagella, $\times 800 .(f)$ Wild-type, $\times 640 .(g)$ Longflagellum mutant $l f I, \times 640$. 

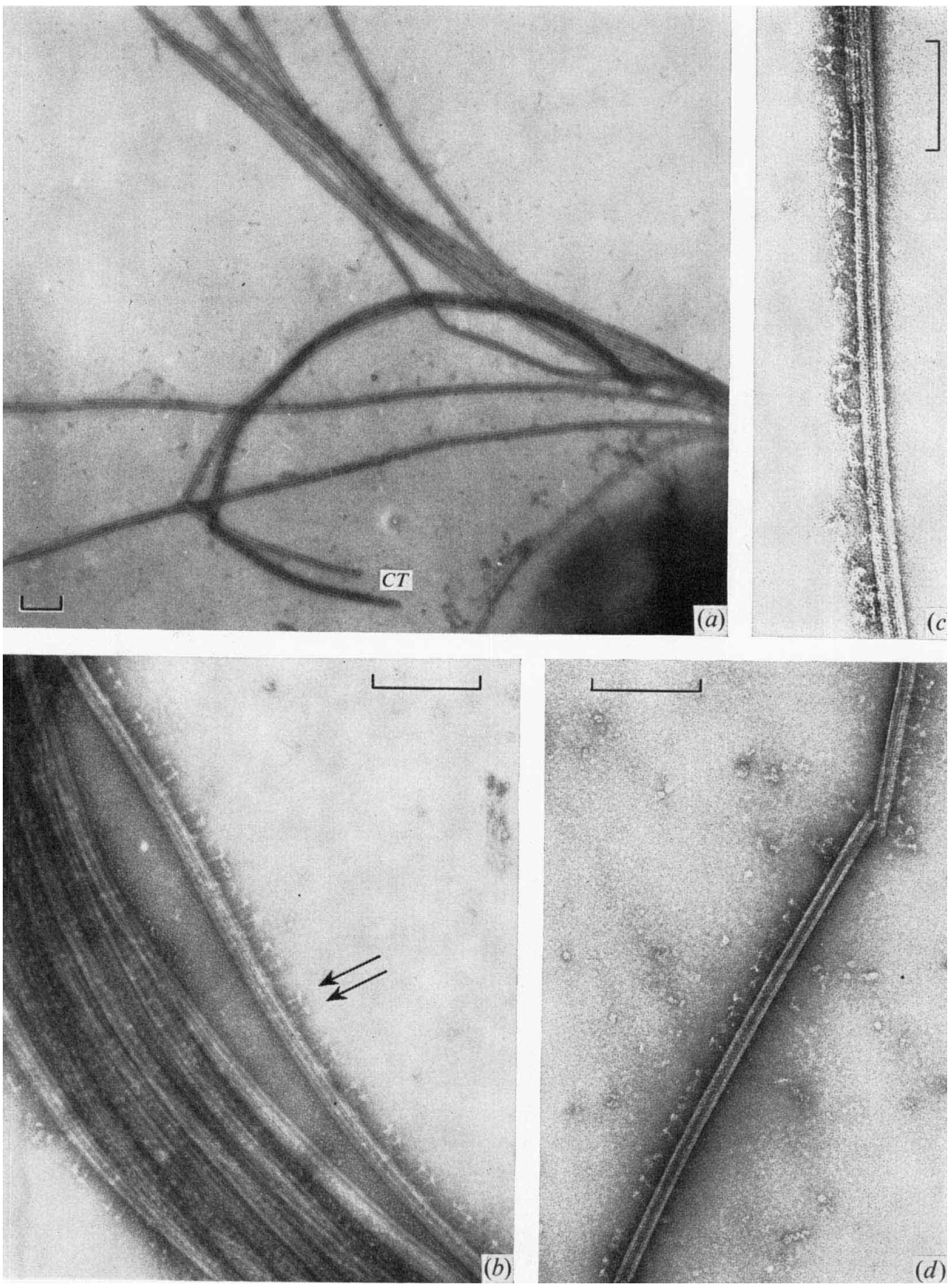

Fig. 4. Electron micrographs of negatively stained frayed flagella. Scale marks represent $0 \cdot 2 \mu \mathrm{m}$. (a) Short-flagellum mutant $p f 7 A$; nine outer doublet tubules and two central tubules $(C T)$ with attached rungs. $(b)$ Wild-type; doublet tubule with spokes (arrows) arranged in pairs. $(c)$ and $(d)$ Short-flagellum mutant $\operatorname{ff}_{7} A$ showing irregularly spaced spokes. 


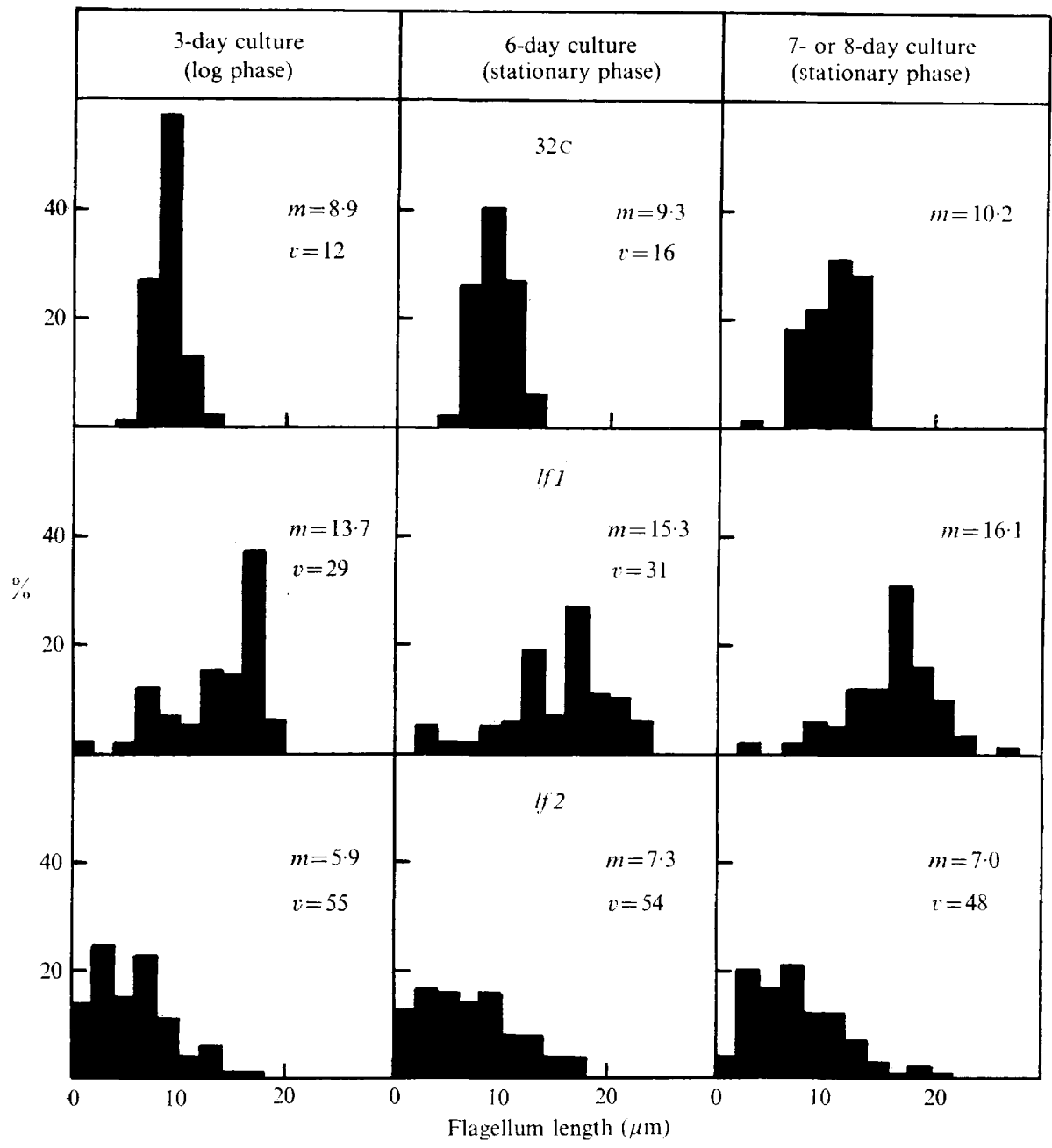

Fig. 5. Distribution of flagellum lengths in cultures of $32 \mathrm{C}$, lf $\mathrm{I}$ and $l f 2$. The sample sizes were 50 organisms ( 100 flagella) for $32 \mathrm{C}$ and $l f_{I}$; for $l f_{2}$ they were 42,53 and 68 organisms $(84,106$ and 136 flagella) for the 3,6 and 8 -day cultures respectively. $m=$ Mean flagellum length in $\mu \mathrm{m} ; v=$ standard deviation expressed as a percentage of the mean.

normal cross-section of the wild-type shown in Fig. $3 b$ ), and central tubules were sometimes absent (Fig. $3 d$ ). Radial spokes were visible in some cross-sections of all strains (Fig. $3 a$ ) but were not seen in some cross-sections of $p f 7, p f 7 A$ and $p f 7 B$. Except in the case of $H A 24$, tubules persisted to the end of the flagellum and at the tip there was a gradual conversion of doublet tubules to singlets as in the wild-type (Ringo, 1967).

Negatively stained preparations of frayed $p f 7 A$ flagella were examined for the presence of two subsidiary flagellar components, the rungs attached to the two central tubules and the spokes attached to the outer tubules (see Hopkins, 1970). Organisms were suspended in NFM and bubbled for 2 or more $h$ to obtain good flagellation before resuspending in T/EDTA. Bubbling in distilled water resulted in much flagellar detachment and breakage during sonication. Optimum results were obtained when cells were left long enough in the T/EDTA solution for most of the flagellar membranes to become swollen before sonicating. 
Shorter times in T/EDTA gave less fraying and longer times resulted in detachment of flagella from the organisms prior to sonication.

Normal rungs were seen in $p f 7 A$ (Fig. $4 a$ ) but the spacing and strength of attachment of the spokes appeared abnormal. It was confirmed that spokes occur in pairs in the wild-type, the intra-pair distance being about $30 \mathrm{~nm}$ and the inter-pair distance about $70 \mathrm{~nm}$ (Hopkins, I970, and Fig. $4 b$ ). In $p f 7 A$ the spokes, although having normal morphology and dimensions, appeared to be irregularly spaced and to detach more readily than in the wild-type (Fig. $4 \mathrm{C}$, $d)$. The spokes in Fig. $4(d)$ show the greatest regularity in spacing and the least detachment yet seen in $p f 7 A$. There was some indication that the spokes might be more closely spaced and hence more numerous in this strain. The mean of 42 inter-spoke distances measured from Fig. $4(c)$ and $(d)$ was $36 \mathrm{~nm}$, giving an average value of 28 spokes $/ \mu \mathrm{m}$ length of tubule compared with $2 \mathrm{I} / \mu \mathrm{m}$ in the wild-type.

Genetic analysis of short-flagellum mutants has identified three loci: $p f 7 A$ and $B$ are probably allelic with $p f 7 ; p f 8 A, B, C$ and $D$ are probably allelic with $p f 8$, and $p f 2 I$ is at a third locus (McVittie, 1972). Repeated attempts to cross $H A 24$ were unsuccessful and no attempts were made to cross $S_{16}$, but since these strains differed phenotypically from each other and from the other strains they probably represent two further loci.

It was clear from the light-microscope observations described above that there was as much intra-locus as inter-locus variation between mutants. Fine-structure studies showed, however, that disorganization of the flagellar shaft (poorly formed, abnormally positioned tubules and abnormal spokes) was most evident in the $p f 7$ mutants, less so in $p f 8$ mutants and least so in $p f 2 r$.

Long-flagellum mutants. Mutants $l_{I}$ and $l f_{2}$, which are at distinct genetic loci (McVittie, I972), were both characterized by the presence of abnormally long flagella (Fig. $3 \mathrm{~g}-$ compare with the wild-type Fig. $3 f$ ). The distributions of flagellum lengths in samples of non-synchronized liquid-grown cells are given in Fig. 5. The mean lengths and coefficients of variation (standard deviation expressed as a percentage of the mean) are also given; data for the wild-type, $32 \mathrm{C}$, are given for comparison. The samples consisted of flagellated cells only. At 3 days the organisms were growing exponentially while the 6-, 7- and 8-day samples represented stationary phase organisms. Data from a second 3-day sample of $32 \mathrm{C}$ and $l f_{I}$ gave histograms very similar to those shown in Fig. 5 .

Flagellum lengths in $32 \mathrm{C}$ were symmetrically distributed with mean values of about 9 to Io $\mu \mathrm{m}$, the mean being slightly higher in stationary phase than in log phase. This could be accounted for by the absence, in stationary phase, of organisms still undergoing postdivision flagellar growth. These organisms accounted for the shoulder on the left-hand side seen only in organisms from the exponential phase (Fig. 5). The maximum flagellum length did not exceed $\mathrm{I} 4 \mu \mathrm{m}$.

Mean flagellum length in $l f I$ was considerably higher than in $32 \mathrm{C}$ and tended to increase with age of culture. In organisms from the exponential phase the length distribution was asymmetrical, the mode being higher than the mean. Values for the coefficient of variation showed that there was much greater variability in length than in $32 \mathrm{C}$ and in addition to the large proportion of abnormally long flagella, i.e. those longer than $14 \mu \mathrm{m}$, there were also more short flagella of less than $6 \mu \mathrm{m}$. Maximum flagellum length rose to 22 to $24 \mu \mathrm{m}$ with occasional flagella 26 to $28 \mu \mathrm{m}$ long, twice the maximum length for $32 \mathrm{C}$.

Although the maximum flagellum length in $l f 2$ was well outside the wild-type range, it was less than in $l f_{\mathrm{I}}$, rising from $\mathrm{I} 6$ to $\mathrm{I} 8 \mu \mathrm{m}$ in the 3 -day sample to 20 to $22 \mu \mathrm{m}$ in the 8 -day sample. The means were lower than in $32 \mathrm{C}$ owing to the large proportion (larger than in both $32 \mathrm{C}$ and $l f_{I}$ ) of rather short flagella. (In a second experiment mean values very similar to 
Table 4. Intra-pair variability in flagellum length in 3-day liquid cultures

of $32 \mathrm{C}$, lf $_{\mathrm{I}}$ and lf $_{2}$, and in lf I $\mathrm{I} 2$ cells in NFM

The sample sizes were 50 cells for $32 \mathrm{C}$ and $l f_{1}, 42$ cells for $l f_{2}$ and 33 cells for $l f_{I} l f_{2}$

\begin{tabular}{|c|c|c|c|c|}
\hline$d / m^{*}$ & $32 \mathrm{C}$ & $l f_{I}$ & $l f 2$ & lfI lf 2 \\
\hline 0.0 to 0.19 & 94 & 72 & 36 & 45 \\
\hline $\begin{array}{l}0.20 \text { to } 0.39 \\
>0.10\end{array}$ & 6 & $\begin{array}{r}20 \\
8\end{array}$ & $\begin{array}{r}7 \\
57\end{array}$ & 6 \\
\hline
\end{tabular}

those for $32 \mathrm{C}$ were obtained.) There were also far fewer abnormally long flagella than in $l f_{I}$ and the variability in flagellum length was greater.

Considerable variability existed between members of a pair of flagella from the same organism in both $l f I$ and $l f 2$. This was determined quantitatively in terms of $d / m$ values where $d$ and $m$ were the intra-pair difference in length and the mean length respectively (see Table 4).

When organisms of $32 \mathrm{C}$, lf $I$ and $l f 2$ were removed from growth medium during exponential growth and resuspended in NFM, flagellum length increased to values similar (or slightly higher in the case of $32 \mathrm{C}$ ) to those obtained in the 6- to 8-day cultures. Thus the increase in length in older cultures may have been due, in part, to nitrogen depletion of the medium.

Cultures of the double mutant $l f_{I}$ lf 2 usually had few flagellated cells. Measurements of flagellum length made on organisms resuspended in NFM showed similar characteristics to those seen in cultures of $l f 2$ (Fig. 5 and Table 4). The maximum length was 20 to $22 \mu \mathrm{m}$ and the mean in a sample of 33 flagellated organisms was $9.7 \mu \mathrm{m}$ with a coefficient of variation of $48 \%$.

The two long-flagellum mutants differed markedly from $32 \mathrm{C}$ in their flagellar regeneration characteristics following artificial deflagellation. Algae used for regeneration experiments had been left overnight in NFM in an attempt to obtain a homogeneous population with a high proportion of flagellated organisms. For $32 \mathrm{C}$ it was possible to obtain 98 to $100 \%$ flagellated cells, while for $l f_{I}$ values of 83,62 and 76 were obtained in different experiments. Deflagellation was complete with no killing, as inferred from the fact that at the end of regeneration the percentage of flagellated organisms was restored to the initial value.

The two main characteristics of flagellar regeneration in $l f_{I}$ were long and variable lag times before measurable growth occurred and a slow elongation rate. Three experiments with $32 \mathrm{C}$ gave over $96 \%$ flagellated cells within $30 \mathrm{~min}$ of deflagellation whereas in If values of only $44 \%, 27 \%$ and 10\% were obtained after $2 \mathrm{~h}$. The values continued to rise until the end of the experiment. In one experiment which was carried on for $30 \mathrm{~h}$ some cells had a lag time of more than $27 \mathrm{~h}$. Owing to the asynchrony of regeneration no information on the kinetics of elongation of individual flagella could be obtained from a plot of mean flagellum length against time. An attempt was made to estimate the maximum elongation rate by plotting the maximum length in each sample against time. The flagella measured were presumably those which had the shortest lag times and were growing fastest. The estimated maximum rate was about $4 \mu \mathrm{m} / \mathrm{h}$ over the initial $6 \mathrm{~h}$ of regeneration. This is still lower than the wild-type rate.

Flagellar regeneration in $l f_{2}$ resembled that in $l f_{I}$ in its main features (Fig. 6). The relationship between mean flagellum length and time was linear during the first $13 \mathrm{~h}$ and extrapola- 


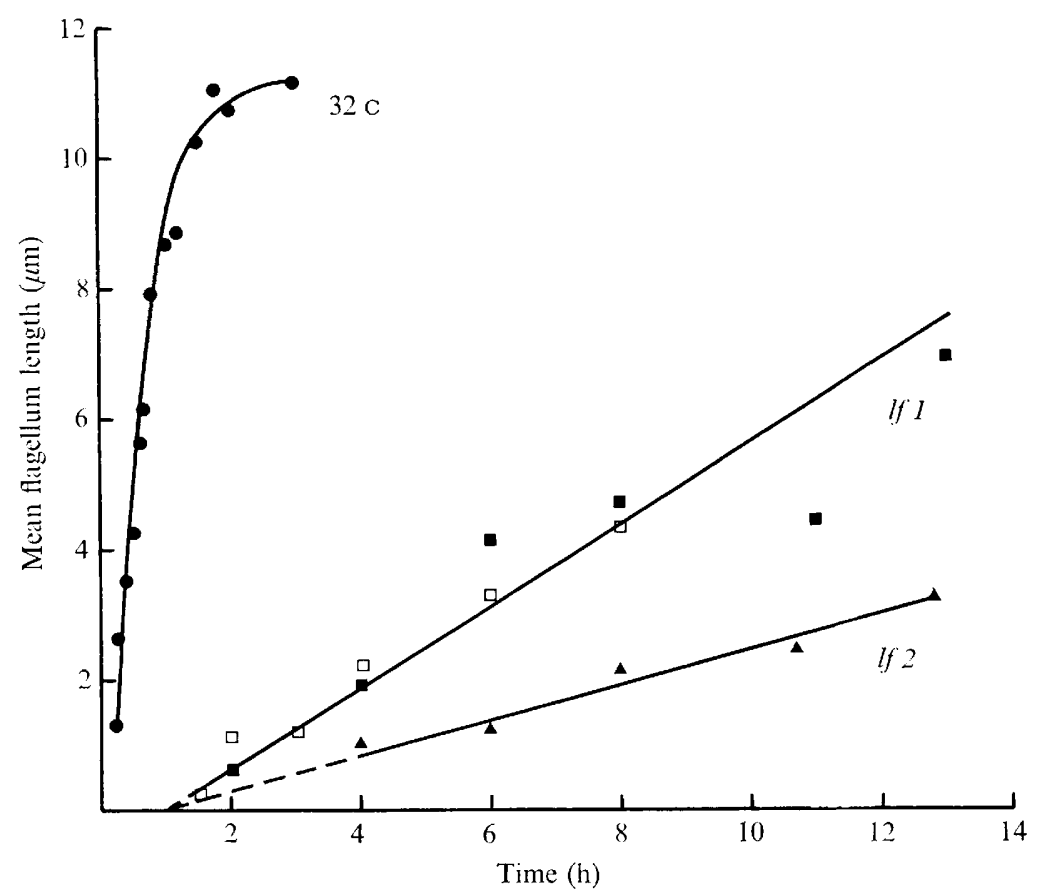

Fig. 6. Flagellar regeneration in $32 \mathrm{C}, l f t$ and $l f 2$. Solid and open squares represent different experiments. Data for $32 \mathrm{C}$ and $l f_{1}$ from Randall et al. (I967).

tion to zero length gave a minimum lag time of about $\mathrm{I} h$; the elongation rate was $0.28 \mu \mathrm{m} / \mathrm{h}$ compared with a rate of $0.63 \mu \mathrm{m} / \mathrm{h}$ in lf (Randall et al. 1967 ). The slower elongation rate could be partly (or perhaps wholly) accounted for by the fact that the rate of increase in the percentage of flagellated organisms was even slower than in $l f I$. At $4 \mathrm{~h}$ only $\mathrm{I} 6 \%$ of organisms were flagellated; the equivalent values for $l f I$ were 48,46 and $32 \%$ in different experiments. As in $l f I$, some organisms had lag times longer than $27 \mathrm{~h}$. During regeneration many organisms had two flagella of grossly unequal length; measurements on 1 I 6 organisms gave $23 \%$ with $d / m$ values of less than $0.20, \mathrm{I} 2 \%$ with values of 0.20 to 0.39 and $65 \%$ with values of 0.40 or more. The frequency of uniflagellate organisms, measured from a sample of 500 algae at $6 \mathrm{~h}$, was only $3 \%$. Thus intra-pair differences in flagellum length must have been due mainly to intra-pair differences in elongation rate rather than in lag time since the latter would yield uniflagellate algae.

The flagella of $l f I$ and $l f z$ tended to be in continuous motion and swimming was seen, although this was usually slower and more jerky than in wild-type; the abnormally long flagella appeared to hamper normal swimming. Colonies of $l f_{I}$ were sometimes difficult to distinguish from wild-type on semisolid agar. It was thus fortuitous that $l f r$ originally arose in a double mutant strain whose colonies could be readily distinguished from wild-type.

Both $l f_{I}$ and $l f_{2}$ had morphologically normal flagella except that the tips were slightly swollen and had a bulbous appearance (Fig. $3 g$ and Randall et al. 1967). The bulbous tips were more pronounced in $l f_{2}$ than in $l f_{I}$ and were also present in $l f_{I}$ lf 2 . Sections showed that the arrangement of tubules was normal and that the space between outer tubules and membrane in the tip region was filled with electron-dense material.

The double mutant $l f_{I} p f_{7}$ had mainly stumps with a few short flagella up to $2 \mu \mathrm{m}$ long. 


\section{DISCUSSION}

The high mutagenic efficiency of NG enabled mutants to be isolated readily without the enrichment step previously used (Warr et al. I966). It also resulted in the production of some double mutants. Some double (or multiple) mutants may have remained undetected because analysis of only ten tetrads would not have detected two or more very closely linked mutations, and because flagellum-less and stumpy mutants could not be crossed.

The fact that flagellum-less mutants all had normal basal bodies may indicate that mutations affecting the basal body are lethal. Basal bodies may be essential for normal division since they appear to be involved in determining the plane of division at cytokinesis (Johnson \& Porter, I968). The cause of the structural disorganization seen in the transitional region in flagellum-less mutants remains unknown. It may be this disorganization which prevents the development of the flagellar shaft.

Stumpy mutants were blocked at a later stage of flagellar development than flagellum-less mutants. The nature of the electron-dense material which accumulated in the short flagellar shaft is unknown, but one possibility is that it was tubule protein. This material is sometimes organized into sheets (McVittie, I969). Stumpy mutants may be analogous to mutants of bacteriophage $\mathrm{T}_{4}$, in which absence of a factor needed for normal polymerization of head protein leads to the formation of aggregates of this protein which are no longer available for assembly into virus capsids (Laemmli, Beguin \& Gujer-Kellenberger, 1970). Alternatively, the tubule protein itself may be abnormal in these mutants and may tend to polymerize prematurely into sheet-like arrays.

The disorganization of flagellar structure seen in short-flagellum mutants suggests that they too were blocked in flagellar development rather than defective in a mechanism controlling length. In most of these mutants functional disability cannot be attributed to the shortilength of the flagella since the length of some flagella exceeded the minimum necessary for motility in the wild-type. The intra-pair differences in flagellum length in some strains may reflect intra-pair differences in elongation rate or in regression rate.

Some of the short-flagellum mutants, particularly those at the $p f 7$ locus, appeared to be abnormal in the attachment and spacing of the spokes. This in turn could reflect abnormalities in the structure of the outer tubules since attachment of the spokes is presumably related in some definite way to tubule substructure. The waviness of the tubules, also noted in newly formed flagellar tubules in Tetramitus (Outka \& Kluss, 1967), may also indicate some abnormality in tubule structure.

Before discussing the long-flagellum mutants, some general comments must be made about the measurement of flagellum length. The length measured was probably slightly shorter than the true length since part of the flagellar shaft was obscured by the wall collar through which the flagellum passes to the exterior. This error would not, however, have been greater than about $0.3 \mu \mathrm{m}$. Electron micrographs have shown that in the wild-type deflagellation results in breakage at the distal end of the transitional region; subsequent outgrowth of the new; flagellar shaft occurs immediately with little or no lag (Randall, 1969; Rosenbaum, Moulder \& Ringo, 1969). The lag time described in this paper refers to the period during which flagella cannot be seen in the light microscope.

The two long-flagellum mutants were clearly defective in a control mechanism which must have operated in the wild-type to limit flagellar growth to a fixed length. Regeneration studies have shown that breakdown in length control is associated with breakdown in the control of elongation rate. This control seems to operate within the flagellum itself since regenerating cultures had large proportions of organisms with two grossly unequal flagella. 
On the other hand, the factor(s) determining the lag time apparently operated within the organism since uniflagellate organisms were rarely seen. Variations in lag time may have been due to variations in the level of intracellular pools of flagellar precursors.

Studies with double mutants indicated that $l f 2$ and the short-flagellum mutant $p f 7$ were both epistatic to $l f I$. The phenotype of $l f_{I} p f 7$ agreed with the suggestion that $p f 7$ and other short-flagellum mutants were blocked in development; without the capacity for normal flagellar development, the potentiality for growth to an abnormal length conferred by the $l f I$ mutation could not be expressed.

It will be apparent that none of the mutants described here has been fully characterized. Other approaches will be needed to achieve this. Some progress has already been made in the chemical analysis of wild-type flagella of Chlamydomonas reinhardii (Jacobs \& McVittie, I970) and a similar analysis of the mutants should reveal any abnormalities in their flagellar proteins.

Some of the work reported here was included in a Ph.D. thesis submitted to the University of London in 1969. I am grateful to Professor Sir John Randall, F.R.S., for support and encouragement, to Dr J. R. Warr for useful discussion, and to Professor D. A. Hopwood for helpful criticism of the manuscript.

\section{REFERENCES}

Dippell, R. V. (1968). The development of basal bodies in Paramaecium. Proceedings of the National Academy of Sciences of the United States of America 6r, 46I-468.

Ebersold, W. T., Levine, R. P., Levine, E. E. \& Olmsted, M. A. (1962). Linkage maps in Chlamydomonas reinhardii. Genetics 47, $53 \mathrm{I}-543$.

Hopkins, J. M. (1970). Subsidiary components of the flagella of Chlamydomonas reinhardit. Journal of Cell Science 7, 823-839.

JACOBS, M. \& MCVITTIE, A. (1970). Identification of the flagellar proteins of Chlamydomonas reinhardii. Experimental Cell Research 63, 53-6r.

Johnson, U. G. \& Porter, K. R. (I968). Fine structure of cell division in Chlamydomonas reinhardii. Journal of Cell Biology 38, 403-405.

LAemmli, U. K., Beguin, F. \& GuJer-Kellenberger, G. (1970). A factor preventing the major head protein of bacteriophage $\mathrm{T}_{4}$ from random aggregation. Journal of Molecular Biology 47, 69-85.

LuFT, J. H. (196I). Improvements in epoxy resin embedding methods. Journal of Biophysical and Biochemical Cytology 9, 409-4I4.

MCVitTIE, A. (1969). Studies on flagella-less, stumpy and short-flagellum mutants of Chlamydomonas reinhardii. Proceedings of the Royal Society B I73, 59-60.

McVitTIE, A. (1972). Genetic studies on flagellum mutants of Chlamydomonas reinhardii. Genetical Research (In the press.)

OutKA, D. E. \& Kluss, B. C. (1967). The amoeba-to-flagellate transformation in Tetramitus rostratus. II. Microtubular morphogenesis. Journal of Cell Biology 35, 323-346.

RaNDall, J. T. (1969). The flagellar apparatus as a model organelle for the study of growth and morphopoiesis. Proceedings of the Royal Society $\mathrm{B}$ I73, 3I-58.

Randall, J. T., Cavalier-Smith, T., McVittie, A., Warr, J. R. \& Hopkins, J. M. (1967). Developmental and control processes in the basal bodies and flagella of Chlamydomonas reinhardii. In Control Mechanisms in Developmental Processes. 26th Symposium of the Society for Developmental Biology. New York and London: Academic Press.

Randall, J. T., WARR, J. R., Hopkins, J. M. \& MCVitTiE, A. (I964). A single-gene mutation of Chlamydomonas reinhardii affecting motility: a genetic and electron microscope study. Nature, London 203, 9I 2-9I4.

RiNGo, D. L. (1967). Flagellar motion and fine structure of the flagellar apparatus in Chlamydomonas. Journal of Cell Biology 33, 543-57I.

Rosenbaum, J. L., Moulder, J. E. \& Ringo, D. L. (I969). Flagellar elongation and shortening in Chlamydomonas. The use of cycloheximide and colchicine to study the synthesis and assembly of flagellar proteins. Journal of Cell Biology 4r, 600-6I9.

WarR, J. R., McVittie, A., Randall, J. T. \& Hopkins, J. M. (I966). Genetic control of flagellar structure in Chlamydomonas reinhardii. Genetical Research 7, 335-35I. 